\title{
Beiträge zur Morphologie der Keimung von Salvinia natans.
}

Von W. Arnoldi.

(Aus dem Botanischen Institute der Universität Charkow.)

(Mit 47 Abbildungen im Text.)

Pringsheim's Beobachtungen an Salvinia natans und ihrer Entwicklungsgeschichte ergaben als Resultat ein allgemeines Bild der Keimung von Makro- und Mikrosporen und der Embryoentwicklung bei diesem Vertreter der heterosporen Farne. Diese Arbeit diente nun als Ausgangspunkt für eine ganze Reihe nächstfolgender Beobachtungen, sowie für ältere, also auch für neuere Gelehrte, welche moderne Versuchsmethoden anwandten. Die Arbeiten Campbell's, hauptsächlich aber Belajeff's, verbesserten bedeutend die Vorstellungen Pringsheim's, insofern sie die Keimung der Mikrosporen und die Entwicklung aus ihnen männlicher Prothallien anbelangten. Jene Gelehrten konnten nicht nur Schritt für Schritt den Vorgang der Mikrosporenkeimung verfolgen, es gelang ihnen auch morphologisch richtig das reduzierte männliche Prothallium aufzufassen. Die Entstehung der Antherozoiden und die histologische Veränderung der spermatogenen Zellen lagen außerhalb der Aufgabe der beiden Botaniker.

Die Makrosporenkeimung und das spätere Schicksal der weiblichen Prothallien war weit weniger eingehend behandelt als die Keimung der Mikrosporen. Nach Pringsheim wurden zwar Untersuchungen an der Makrosporenkeimung vorgenommen, sie führten jedoch zu keinem endgültigen Resultate und Campbell ist deshalb berechtigt auf der 398. Seite der zweiten Auflage seiner „Mosses and Ferns" so sich zu äußern: „... but none of these observers (Pringsheim, Prantl, Arcangeli) were able to follow accurately the earliest divisions in the germinating macrospores".

Da ich die Möglichkeit hatte frisches Material von Salvinia natans aus dem Donetzfluß bei Tschuguew, nicht weit von Charkow, zu beziehen, so beschloß ich den Vorgang der Keimung von beiderlei Sporen noch einmal zu durchforschen, um den dunklen Seiten desselben besondere Aufmerksamkeit zu schenken. Auch interessierte mich in den Beziehungen zwischen Makrospore, Prothallium und Embryo ins Reine zu kommen. Dementsprechende Versuche gaben einige Aufklärung, obgleich die Prothallien sich als ziemlich konstante Bildungen erwiesen und nur nach vieler Mühe sich dem Experimente fügten. 
Mit großer Anerkennung erwähne ich hier meines Schülers, des Studenten N. A. Fedtschenko, welcher eine Reihe von den hierzu beigelegten Zeichnungen ausführte und bei dem Experimentieren behilflich war $^{1}$ ).

\section{Keimung der Mikrosporen.}

Beobachtungen an keimenden Mikrosporen stimmen vollkommen mit Belajeff's Angaben. Deshalb erörtere ich nur noch einige histologische Details.

Die noch ungeteilte Mikrospore, die in der Fig. 1 abgebildet ist, ist durch ein an Öl reiches Protoplasma ausgezeichnet und einen die Mitte der Zelle einnehmenden Kern. Im Kern sind 1-2 Nukleoli zu
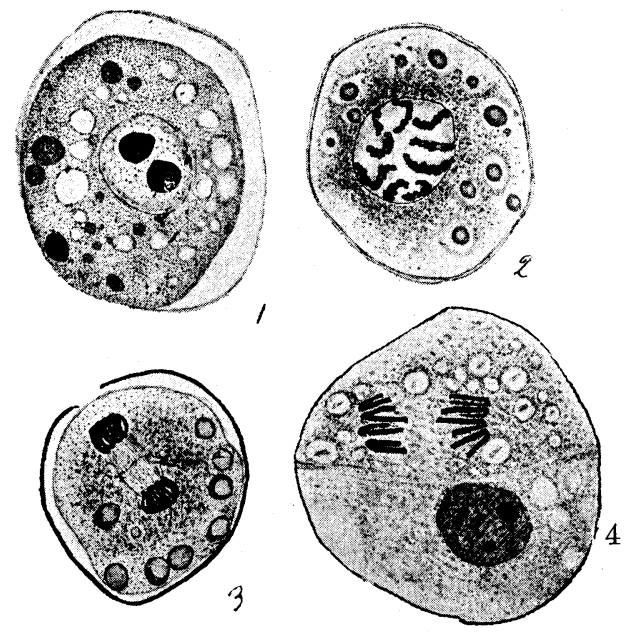

Fig. 1-4. Schnitt durch die Mikrospore vor ihrer Keimung. Vergr. 1500. Fig. 2. Der Kern vor der Tellung; es sind die Chromosomen zu sehen. Fig. 3. Bildung der Tochterkerne. - Fig. 4. Zweite Teilung der Mikrospore.

sehen, ein Chromatinnetz ist nicht bemerkbar. Der Durchmesser des · Kerns schwankt in engen Grenzen und beträgt im allgemeinen $8 \mu$. Wenn sich der Zellkern zur Teilung anschickt, so bilden sich allmählich 4 Chromosomen aus, welche sich der Länge nach teilend $4+4$ Tochterchromosomen geben, und zum Aufbau von 2 neuen Kernen mit je 4 Chromosomen dienen.

Die Zeichnungen 2 und 3 zeigen diese Phasen der Tochterkernbildung und $\mathrm{zu}$ gleicher Zeit die erste Teilung der Mikrospore. Jede Chromosome zeigt dabei eine Länge von $8 \mu$, bei $1 \mu$ Breite. Der Abstand zwischen den Polen der Tochterzellen (Fig. 3) ist $9-10 \mu$

1) Die Abbildungen sind von Präparaten abgezeichnet, welche mittels Flemming's oder Kaiser's Lösung fixiert waren. Als Färbemittel diente Heidenhain's Eisenhämatoxylin oder das dreifache Flemming'sche. 
gleich; die Zellplatte wird $4,5-5 \mu$ weit von jedem Pole angelegt. Dieser ersten Teilung folgt eine zweite, welche $\mathrm{zu}$ einer Spaltung der Mikrospore in 3 Zellen führt. Entweder geht diese Teilung in einer Fläche vor sich, die jener der ersten Teilung parallel steht, die Spore in 3 aufeinander stehende Zellen scheidend, wie aus der Fig. 1 der Belajeff'schen und aus Fig. 5 der vorliegenden Arbeit ersichtlich ist; oder auch nimmt die Kernspindel eine gegen die erste Scheidewand senkrecht verlaufende Fläche ein; diesen Fall habe ich einmal beobachtet (Zeichnung 4). Bei der zweiten Teilung tritt natürlich dieselbe Chromosomenzahl auf, wie aus der beigelegten Zeichnung zu sehen ist, auch ist ihre Länge gleichfalls gleich $8 \mu$ und der Durchmesser gleich $1 \mu$. Bei den nächstfolgenden Teilungen treten ungleiche Kerne auf.

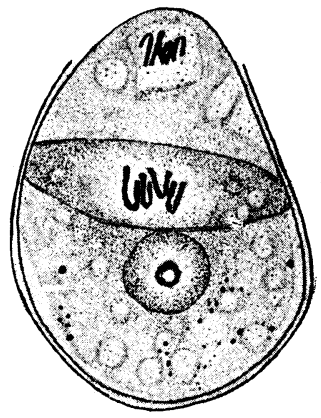

5

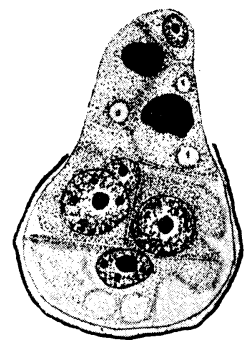

6

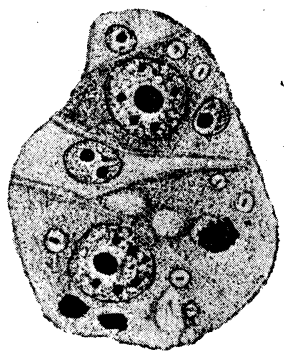

7

Fig. 5. Die zweite Teilung der Mikrospore. Die Spore in drei übereinanderstehende Zellen zerfallen. Vergr. 1000.

Fig. 6-7. Vergr. 1500. Fig. 6. Bildung der Antheridien-Mutterzellen. - Fig. 7 Die Antheridien bilden Deckzellen.

Einige von ihnen behaupten hartnäckig die frühere Größe, nämlich $8 \mu$ im Durchmesser, die übrigen aber werden bedeutend kleiner. Die großen Kerne werden nur von den generativen Zellen beibehalten, die kleineren kommen für die vegetativen, Scheitel- und AntheridiumWandzellen, sowie für die mittlere sterile Zelle in Betracht $(B, K$, Krn II, siehe Belajeff's Arbeit Taf. I, Fig. 6 und 7). Fig. 6 der vorliegenden Arbeit entspricht dem in Fig. 3 des Belajeff'schen Werks abgebildeten Stadium.

Den unteren Teil der Zeichnung nimmt die große untere sterile Zelle mit großem Kern ein, ihr liegt unmittelbar die generative Zelle an, deren Kern sich im Spiremstadium befindet; als Resultat der Teilung geht die Antheridiumdeckzelle, sowie spermatogene Zelle hervor. Höher befindet sich die mittlere sterile Zelle, über welche das zweite 
Antheridium mit seiner Deckzelle liegt; den obersten Teil der Mikrospore nimmt die Scheitelzelle ein. Dies Präparat zeigt deutlich die Ungleichheit der Zellkerne in den verschiedenen Zellen. Fig. 7, die einen ferneren Schritt in der Keimung der Mikrosporen abbildet, zeigt, daß̉ auch der Zellkern der unteren vegetativen Zelle den generativen Kernen in Größe nachsteht. Jede generative Zelle des Antheridiums teilt sich bald nach Abteilung der Deckzelle in zwei Zellen, wie aus Fig. $8 \mathrm{zu}$ sehen ist. Auch in diesem Falle bleibt die Größe der Chromosomen gleich $8 \times 1 \mu$. Von Zentrosomen ist auf diesem Stadium noch keine Spur zu finden. In einem Falle, wie Fig. 9, ist z. B. die beste Gelegenheit gegeben ein Zentrosom zu beobachten, doch ist ein solches nicht vorhanden. Nach der ersten Teilung der generativen Zelle findet deren zweite Teilung in vier Zellen statt. Noch vor der Kernteilung kann man zu beiden Seiten des Kerns je ein Zentrosom

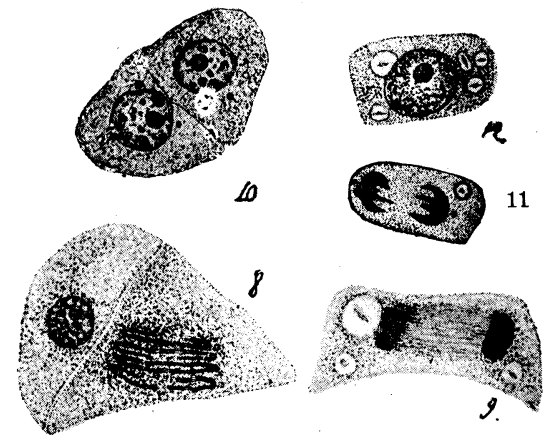

Fig. 8-12. Fig. 8. Der Kern der Antheridium - Mutterzelle teilt sich. - Fig. 9. Der Kern der spermagenen Zelle teilt sich. - Fig. 10. Spermazyten zweiter Ordnung mit zwei Zentrosomen. - Fig. 11. Teilung des primären Spermazyten. -

Fig. 12. Spermazyt. Vergr. 1500.

erblicken, welchen Fall Fig. 10 illustriert. Diese Figur entspricht den Fig. 4 und 7 von Belajeff (II), Fig. 7-11 von Shaw, Fig. 14 von Jamanouchi und Fig. 79 von Campbell (II). Shaw nennt diese Zellen Spermatocyten zweiter Ordnung, Belajeff (II) SpermatozoidenUrmutterzelle, Campbell legt ihnen keine besondere Benennung bei, und spricht einfach von "spermagenetic cell, before the final division". Das Hauptinteresse dieses Stadiums besteht im Erscheinen der Zentrosomen oder künftigen Blepharoplasten, deren Ursprung noch immer unklar bleibt. Nur in der Arbeit Iken o's (I) über die Spermatogenesis bei Marchantia findet man positive Angaben über die Entstehung der Zentrosomen aus den Kernen der spermagenen Zellen (Fig. 1-3 der Arbeit Ikeno's). Übrigens bestreitet Escoyez (I), der neueste Forscher der Blepharoplastenbildung, die Ansicht I ken o's. Andere Autoren fangen in ihren Beobachtungen mit dem Stadium an, welches in meiner Zeichnung abgebildet ist. 
Diese Spermatide zweiter Ordnung teilt sich in zwei Zellen, wie das in Fig. 11 abgebildet ist. Nach der Teilung bekommt man vier Zellen zu sehen, welche man mit Shaw als Spermatiden bezeichnen kann. Jede von ihnen führt ein Zentrosom, welches später sich in den Blepharoplast verwandelt; in Fig. 12 ist so ein Spermatide gleich nach seiner Entstehung zu sehen. Das darauf folgende Schicksal des Spermatiden besteht in seiner Verwandlung in den Körper des Spermatozoiden. Sein Blepharoplast fängt an sich zu verlängern, verschiedene Lagen gegenüber dem Zellkern einnehmend. Die Fig. 13 und 14 stellen die ersten Stadien der Verwandlung des Blepharoplast dar, Fig. 15-17 zeigen die späteren Änderungen des Spermatiden. Fig. 18 und 19 zeigen den Spermatiden von einer anderen Seite, nämlich den

Fig. 13-21. Vergr. 1500. Verwandlung des Spermatiden in den Körper des Antherozoiden. - Fig. 13. Der Blepharoplast, sich etwas verlängernd. - Fig. 14. Die Verlängerung des Blepharoplast geht weiter vor. - Fig. 15-17. Die Kerne verlieren ihre Nukleoli und erscheinen einförmig gebaut. Die Blepharoplasten stark verlängert. - Fig. 18--19. Spermatiden, von hinten gesehen. - Fig. 20. Entwicklung des Körpers des Antherozoiden. Man sieht zahlreiche Cilien, die vom Blepharoplast abgehen. In den Zellen Stärkekörner.

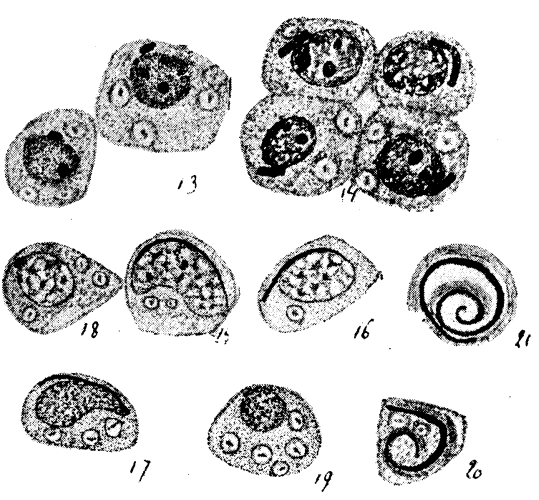

Hinterteil des in die Länge gestreckten Kerns. In den Abbildungen 20 und 21 endlich sieht man den ersten Spermatozoiden, von dessen vorderen Teil zahlreiche Cilien auswachsen, welche an dem den Vorderteil des Spermatozoiden einnehmenden Blepharoplast angeheftet sind. Der Kern steht etwas abseits von dem Vorderrand des Antherozoiden. Fig. 22 stellt einen frei im Wasser herumschwärmenden Antherozoiden dar, nach Behandlung mit Osmiumsäure und Färbung mit Methylgrün + Fuchsin nach den Angaben von Belajeff. Man sieht, wie auffallend lang im Verhältnis zur Körpergröße die Cilien dieses Antherozoiden sind. Eine gewisse Ähnlichkeit hat damit der Antherozoid von Nephrodium, der in Fig. 33 der Arbeit von Jamanouchi abgebildet ist. Die Spermatiden von Salvinia, wie aus den beigelegten Zeichnungen ersichtlich ist, sind durch den Reichtum von großen Stärkekörnern ausgezeichnet. Während der Herausbildung des Antherozoiden sammeln sich die Stärkekörner in einem protoplasmatischen Bläschen an, welches 
am Hinterteil des Antherozoiden hängt. Der Antherozoid entschlüpft aus dem Antheridium in Form eines spiralen Fadens. In solchem $\mathrm{Zu}-$ stande schwimmt er im Wasser herum. Unter dem Einflusse der Fixierung, während des Schwärmens zuweilen und während der Befruchtung stets, verliert der Antherozoid das Bläschen, und dann verändert sich gewöhnlich die Form seines Körpers: die gekrümmte Spirale wird $\mathrm{zu}$ einer ausgebreiteten, wie in der Fig. 22 wiedergegeben ist.

Fig. 22 zeigt, daß der

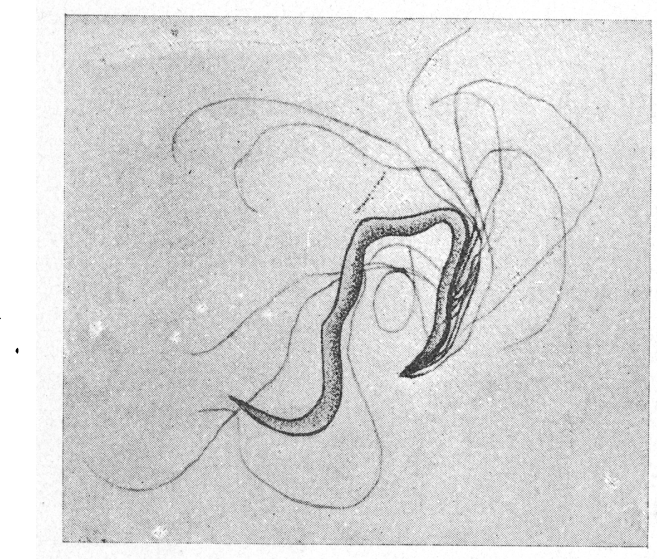

Fig. 22. Antherozoid in Wasser, durch $\mathrm{OsO}_{4}$ getötet und mit Methylgrün + Fuchsin gefärbt. Vergr. 1500.

Kern der Bauchseite des Antherozoiden anliegt, während die Rückseite in einen Kamm mündet, dessen Vorderteil vom Blepharoplast mit den $\mathrm{Ci}$ lien eingenommen wird:Im Hinterteil gelingt es einen Teil des Protoplastes, der frei vom Zellkern ist, gewahr zu werden. Um diese Antherozoidentwicklung zu schließen, muß ich noch auf einen Punkt aufmerksam machen, den ich nicht vermochte klar zu stellen. I Ich konnte nämlich nichts von dem, was Ikeno bei Marchantia als Nehenkörper bezeichnet, finden; eine ähnliche Bildung beschrieb auch Bolleter (I) bei Fegatella conica (Fig. 20 der Taf. XII, siehe das.); auch Jamanouchi hatte es mit einem solchen „Nebenkörper“ zu tun. Keiner der Autoren wußte diesen Nebenkörper zu deuten.

Indem ich meine Angaben mit denen anderer Autoren vergleiche, werde ich $\mathrm{zu}$ dem Schlusse gedrängt, daß kein zureichender Grund vorliegt den Blepharoplast mit dem Zentrosom zu identifizieren. Der Blepharoplast ist ja ein ausgesprochenes funktionierendes Organ, das man bei vielen Pflanzen vorfindet, im allgemeinen überall dort, wo Cilien da sind. Eine neuere Beobachtung von Davis an Derbesia und ihren Zoosporen wiesen auch bei dieser Meeresalge einen unzweifelhaften Blepharoplast nach. Was das Zentrosom anbelangt, so ist das Vorhandensein desselben bei den Gefäßkryptogamen und Blütenpflanzen noch nicht konstatiert worden und darum scheint mir die Identifizierung 
des bekannten Organs mit dem noch problematischen Zentrosom gewagt. Ich glaube, der Blepharoplast sei ein Organ sui generis, das vielleicht dann besser erklärt wird, wenn wir sorgfältige Kenntnisse über die verschiedenen Bewegungsorgane im Pflanzenreich besitzen.

\section{Keimung der Makrospore und Entwicklung des weiblichen Prothalliums.}

Über die ersten Stadien der Makrosporenkeimung gibt es in der botanischen Literatur fast gar keine klare Angaben, außer der konfusen Beschreibung Prantl's, welche vollkommen erklärlich ist, wenn man sich erinnert, daß zu jener Zeit die Mikrotomtechnik in die botanische Praxis noch nicht eingeführt war. Die Makrospore ist bekanntlich eine große Zelle. Sie schwimmt im Wasser in einem Panzer, der aus der Sporangiumwand und drei der Zelle selbst angehörenden Hüllen besteht: dem schaumigen Episporium, dem dicken Exosporium und dem zarten Endosporium. Das schwarz gefärbte Exosporium läßt von dem Innern gar nichts sehen. Im oberen Teile der Spore, wo das Episporium durch eine dünne Spalte durchbrochen wird, sammelt sich ein dicht mit kleinen Stärkekörnern angefülltes Plasma an. In der Mitte der Spore befindet sich der Kern mit einem Durchmesser von $20 \mu$, in dessen Innern man viele kleinere Körner

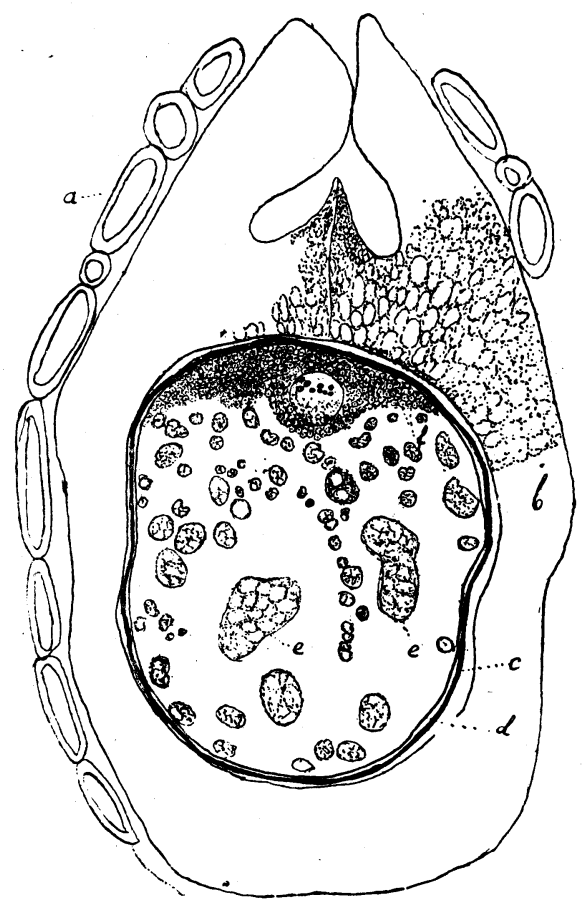

Fig. 23. Schnitt durch eine Makrospore. $a$ Wände des Makrosporangiums; $b$ Episporium ; $c$ Exośporium; $d$ Endosporium; $e$ Proteïnmassen; $f$ Stärkekörner. und ein dünnes Chromatinnetz erblickt. Der übrige Teil der Spore ist durch Proteïnanhäufungen und kleine Stärkekörner besetzt. Fig. 23 stellt einen Schnitt durch solch eine Spore dar. Es ist die allbekannte, in mehrere Lehrbücher aufgenommene Zeichnung Strasburger's; nur die kleinen Stärkekörner fehlen bei Strasburger's Abbildung. Fig. 24 
stellt bei stärkerer Vergrößerung den Vorderteil des Sporenprotoplasmas dar, in dem außer dem Kern noch größere und kleinere Stärkekörner zu sehen sind. Der Kern der Spore verdoppelt sich durch Teilung, doch ist eine Zellwand zwischen den Tochterkernen nicht $\mathrm{zu}$ bemerken. In

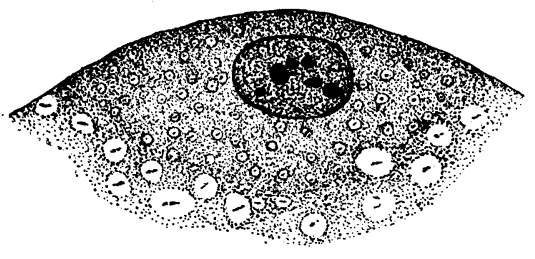

Fig. 24. Der obere Teil des Protoplastes der Makrospore. Kern und Stärkekörner. Vergr. 1000.

Fig. 25-27. Bildung des Gewebes des weiblichen Prothalliums.

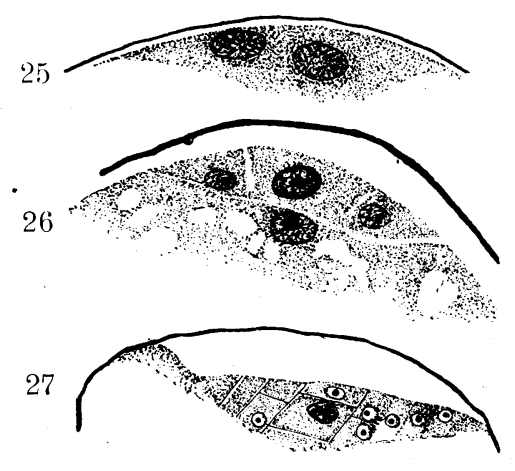

Fig. 25 haben wir eine zweikernige Spore. Danach vermehrt sich die Zahl der Kerne und dann haben wir das Stadium von Fig. 26: drei kleinere in Zellen zerfallene Spore, die nichts anderes als die ersten Zellen des Prothalliums darstellen, und eine vierte größere Zelle, welche den ganzen übrigen Teil der Spore einnimmt. Fig. 27 ist ein weiteres Stadium und zeigt den allmählichen Aufbau des Prothalliums.

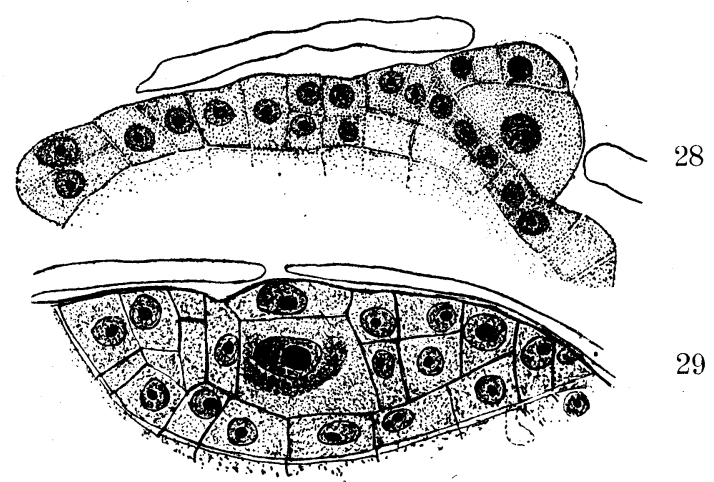

Fig. 28 u. 29. Weitere Ausbildung des Prothalliumgewebes. Die Mutterzelle des Archegoniums ist be1 sonders bemerkbar. Vergr. 500.

Dieser Prozeß ist sehr ähnlich dem, was ich und andere Forscher an dem weiblichen Prothallium von Selaginella und Isoëtes sahen.

Schon auf diesem früheren Stadium zeichnet sich die mittlere Prothalliumzelle, die künftige Mutterzelle des ersten Archegoniums unter den andern Zellen, durch besondere Größe aus. Dieser Umstand 
ist nicht ohne weitere Bedeutung. Er wirft einiges Licht auf den Charakter des Prothalliums eines anderen heterosporen Farn-Marsilia, dessen vegetatives Gewebe erst nach vorhergehender Befruchtung entsteht. Die darauf folgende Entwicklung des Prothalliums besteht im allmählichen Wachstume und Zellteilung, sowie der Bildung des ersten

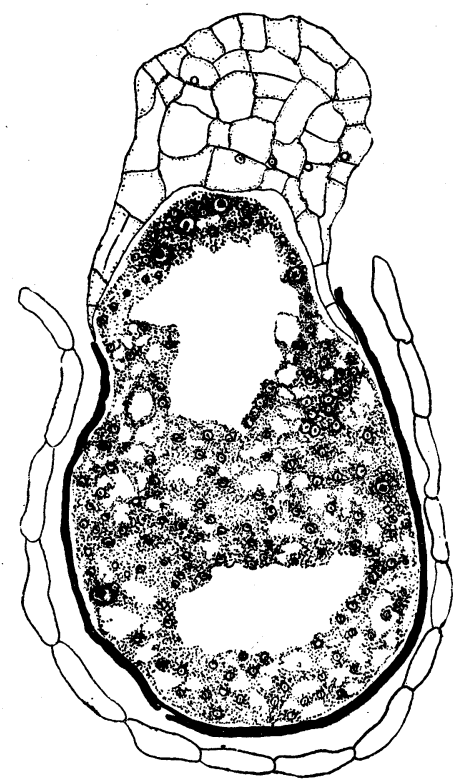

Fig. 30. Schnitt durch die Makrospore mit Prothallium und Kernen im Hohlraum der Makrospore.

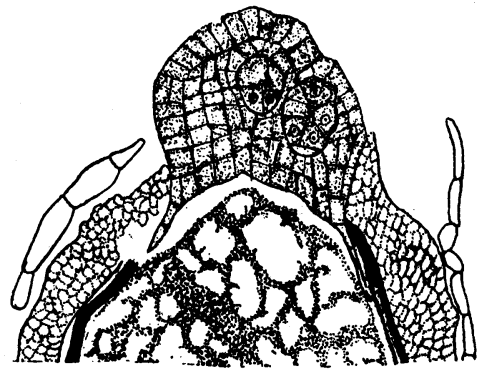

Fig. 31. Junges Prothallium mit zwei Embryos.

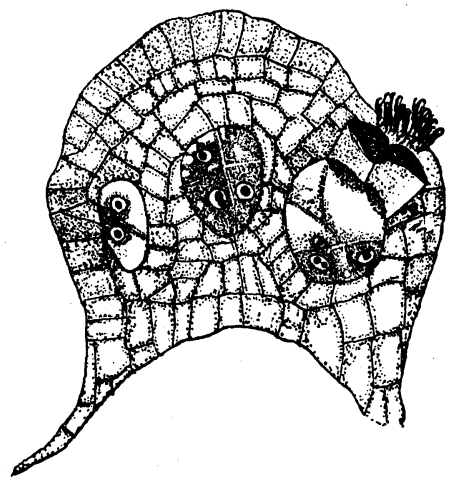

Fig. 32. Junges Prothallium mit drei Embryos.

Archegoniums, welches in diesem Prothallium eine zentrale Lage einnimmt. Fig. 28 zeigt ein Prothallium, dessen Zellgewebe stellenweise zweischichtig, stellenweise auch einschichtig ist; die Archegoniummutterzelle hat ihren Hals noch nicht gebildet. Fig. 29 gibt ein etwas späteres Stadium wieder, nachdem das Prothallium mehrschichtig geworden ist und von der Archegoniummutterzelle eine Halszelle abgeschnitten ist. Darauf, auf Grund von Zunahme des Wachstums und der Vermehrung des Zellkomplexes, bricht die Sporenmembran und das 
Prothallium kommt durch die geborstene Wand zum Vorschein. Diese Stadien sieht man in Fig. 30-32. In der Fig. 30 erkennt man den Hohlraum der Spore, der durch Stärkekörner und Proteïnkörper besetzt ist (letztere sind nicht abgebildet worden) und in dem man deutlich einige Kerne unterscheiden kann; zwei von ihnen sind schon tief hinab zum Grunde der Spore gestiegen.

Während des nächstfolgenden Wachstums des Prothalliums beginnt nach und nach der Unterschied zwischen seinen Seiten hervorzutreten. Diesen Unterschied kannte schon Pringsheim (s. daselbst Taf. XXXI, Fig. 1, Taf. XXIX, Fig. 8); aber besondere Aufmerksamkeit schenkte

33

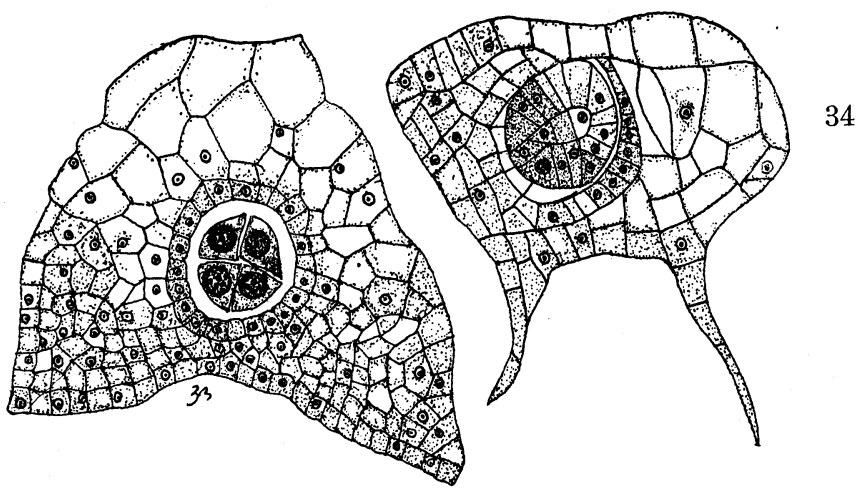

Fig. 33. Prothallium, von oben gesehen. In der Mitte der Embryo. Nur ein Archegonium ausgebildet. Das sterile Drittel sticht vom meristem. Rand ab. Fig. 34. Längsschnitt durch das Prothallium. Unterschied zwischen den Prothallium- und Embryozellen.

er dieser Erscheinung nicht. Etwas umständlicher behandelt das Salvinia $O$ Prothallium Prantl in einer kleinen, aber sehr inhaltsvollen Schrift (ohne Abbildungen). Prantl nennt eine Seite des Prothalliums „sterile Seite“, sie bedingt die Prothalliumseiten; man erkennt eine Vorderseite und zwei Flügel. Diese Verhältnisse sind in Fig. 33 zu überblicken, welche Zeichnung das Prothallium von oben gesehen wiedergibt. Der hintere Teil trägt niemals Archegonien, seine Zellen verlieren sehr bald ihren meristematischen Charakter und erreichen beträchtliche Größe (Fig. 33). Fig. 34 zeigt auf einem Längsschnitt dieselbe Verschiedenheit im Aufbau des Prothalliums - das sterile Drittel, das die rechte Hälfte der Zeichnung einnimmt, besteht aus großen Zellen mit wenig Protoplasma, dagegen ist der vordere Teil des Prothalliums aus kleinen Zellen mit dichtem Prothoplasma aufgebaut. Denselben meristematischen Charakter zeigt der vordere Teil der in 
Fig. 33 abgebildet ist. Ein ähnliches Bild zeigen die Zeichnungen Bauke's, die in Goebel's Organographie aufgeriommen sind. Bei folgendem Wachstum entwickelt das Meristema der Vorderseite und der beiden Seiten flügelartige Vorsprünge, welche längs der Makrosporenmembran herunterlaufen.

Einstweilen wollen wir die Frage über den Zusammenhang zwischen der Form des Prothalliums und den äußeren Bedingungen nicht berühren, wir gehen lieber zur Beschreibung des Baues und der Verteilung der Archegonien über. Schon früher wurde darauf aufmerksam gemacht, wie früh das erste Archegonium angelegt wird, das eine zentrale Lage einnimmt. Fig. 28 zeigt das erste Entwicklungsstadium, die Zelle,

Fig. 35. Allmähliche Entwicklung des Archegoniums. a Randzelle des Prothalliums, zum Archegonium werdend; $b$ ihre erste Teilung; $c$ zweite Teilung; $d$ Eizelle, Hals- und Bauchkanalzelle des fertigen Archegoniums; $e$ dasselbe, Halszelle mit zwei Kernen. Vergr. 500.
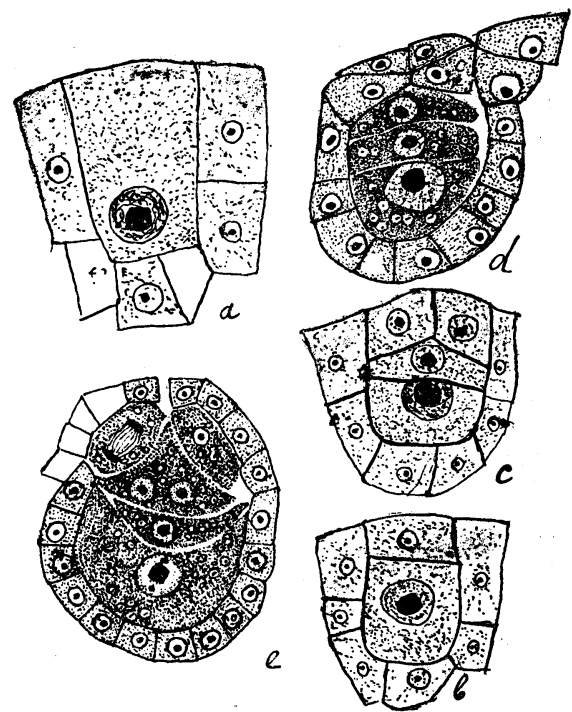

die alș Ausgangspunkt für den Ausbau des Archegoniums dient. Fig. 29 stellt das folgende Stadium dar: die Mutterzelle des Halses hat sich abgeteilt. Fig. $35 a-c$ zeigt, wie das embryonäre einzellige Archegonium alle seine Bestandteile formt, nämlich den Hals, Hals- und Bauchkanalzelle und Eizelle. Die Größe des weiblichen Kerns schwankt zwischen $10 \mu$ (bei der ersten Teilung der Prothalliumsporen) und $20 \mu$ in den Eizellen. Fig. $35 e$ stellt ein ausgewachsenes Archegonium kurz vor der Befruchtung dar; man sieht die keilförmig zwischen die Halszellen sich einschiebende Halskanalzelle. Sie führt zwei Kerne, was nicht selten bei den Gefäßkryptogamen vorkommt. Treub führt ebenso eine zweikernige Halskanalzelle für Lycopodium an, ich beschrieb dieselbe Erscheinung bei Isoëtes. 
Das Protoplasma ist in allen Zellen der Zentralreihe mit kleinen Stärkekörnern angefüllt, die den Zellen des weiblichen Prothalliums von Salvinia so eigen sind. Wie man also sieht, stimmt diese Beschreibung im wesentlichen mit der Pringsheimschen überein; bekanntlich ist gerade bei Salvinia die Hals- und Bauchzelle des Archegoniums zum ersten Male beschrieben worden. Ist das erste Archegonium befruchtet, so hört zuweilen die Ausbildung weiter Archegonien auf. Weit öfter jedoch entstehen außer dem Haupt- und Zentralarchegonium noch zwei

36

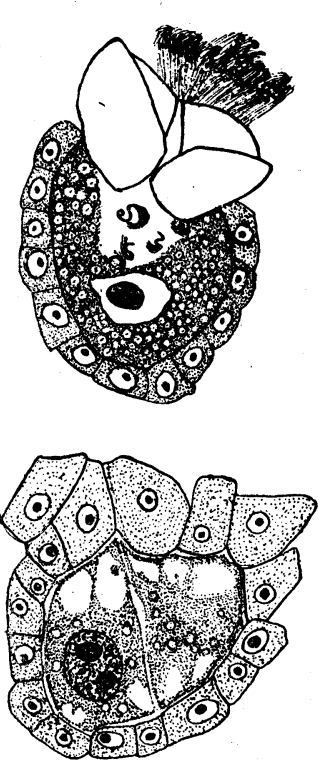

$38 a$

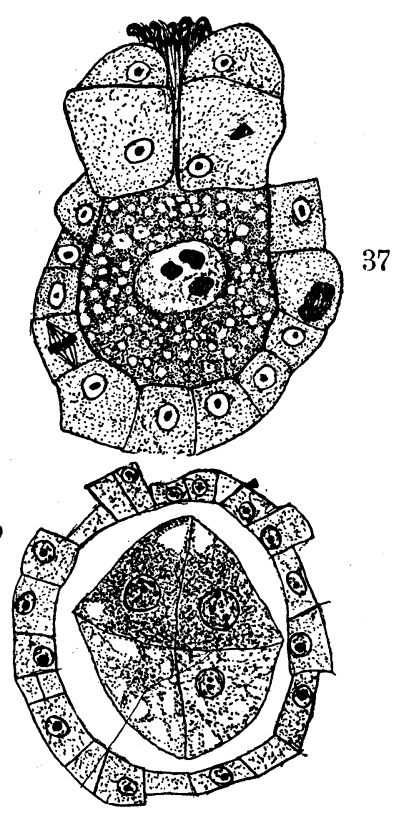

$38 b$
Fig. 36 u. 37 . Antherozoiden, in das Archegonium eindringend.

Eikern mit Antherozoidkerne (Kerne?).

Vergr. 500.

Fig. 38. Erste Teilung des befruchteten Eies. Vier Zellen des Embryos, von oben gesehen; Verschiedenheit in Dichtigkeit des Plasmas und der Größe der Kerne. Vergr. 500.

Nebenarchegonien, das eine links, das andere rechts vom Hauptarchegonium, wie schon Pringsheim und Bauke zeigten. Im Falle aber, wenn die Befruchtung nicht zustande kommt, entsteht eine große Anzahl von Archegonien, was auch schon von Pringsheim und Bauke bemerkt worden ist. Später komme ich noch zu diesem Punkte zurück. Wenn das Archegonium reif genug ist, so verschleimen die Bauchund Halskanalzelle und öffnen den Kanal, durch den die Antherozoiden in das Archegonium hereinschwärmen. Fig. 36 stellt so ein Archegonium im Moment der Befruchtung dar. Der vordere Teil des Eiprotoplasmas ist stark eingedrückt. In diesem Hohlraume befinden sich zahlreiche Antherozoiden und noch mehr derselben sind beim Eingang zum 
Archegonium anzutreffen. Der Eikern liegt in der Nähe des Hohlraums Ich umgehe einstweilen die genaueren Umstände der Befruchtung, da ich noch nicht eine genügende Serie von Stadien besitze. Die neuesten Untersuchungen Yamanouchi's über die Befruchtung von Nephrodium und die frühere Beobachtung von Shaw an Onoclea zeigen, daß wäh-

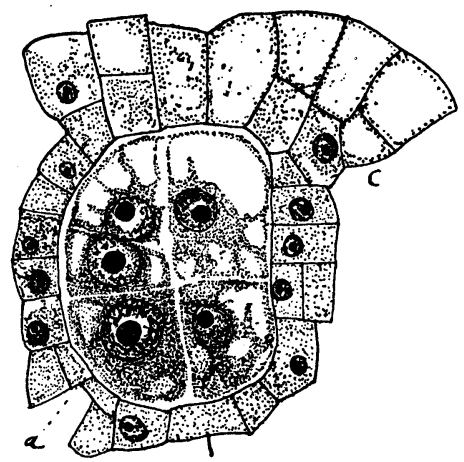

39

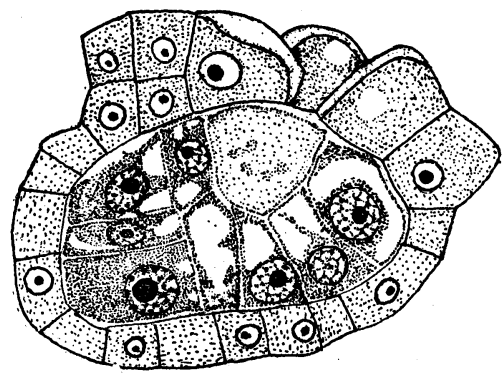

40

Fig. 39-40. Weitere Entwicklung des Embryos im Längsschnitt. Zwei Teile differenziert: der künftige Fuß und der künftige Sproß. Vergr. 1000.

rend der Befruchtung bei den Farnen der männliche Kern in den weiblichen Kern aufgenommen wird und längere Zeit darin seine Selbständigkeit bewahrt (vgl. Yamanouchi; Plate VIII, Fig. $51-\tilde{5}$ s s). Fig. 37 kann man deshalb als ein solches Stadium auffassen, während welchem der Antherozoidenkern bereits in den Eikern eingetreten ist, wobei aber sein Chromatingehalt noch

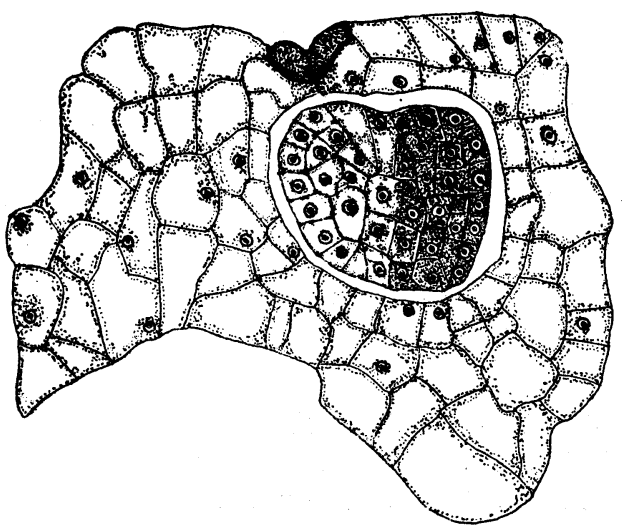

Fig. 41. Späteres Stadium des Embryos als das in Fig. 39-40. Vergr. 250. nicht gleichmäßig über den weiblichen Kern verteilt ist. Nach der Befruchtung geht der Eikern der inzwischen eine Zellhaut gebildet hat, zur Teilung über.

In Fig. $39 a$ ist das erste Stadium dieser Teilung abgezeichnet, während welchem der Eikern in zwei Hälften zerfällt. Die erste Tei- 
lungsfläche geht durch die Vertikalachse des Archegoniums. Die zwei nächsten Teilungen zerschneiden den Embryo in acht Oktanten, wie man aus der Fig. 32 (die Mitte des Embryos darstellend) und Fig. 33 und $38 b$, wo der Embryo von oben gesehen abgebildet ist, sieht.

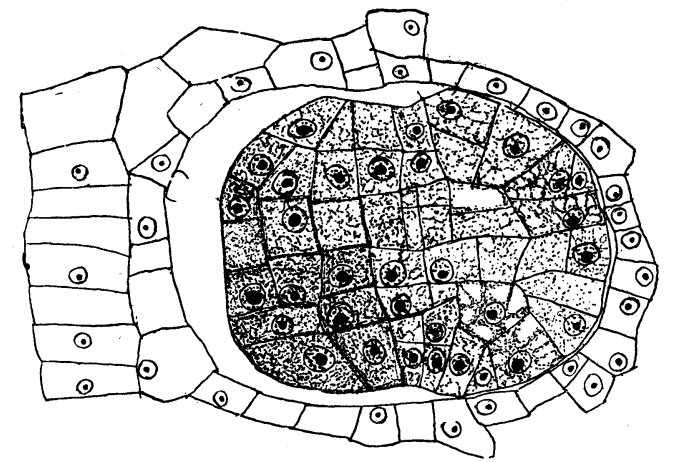

Fig. 42. Querschnitt durch den Embryo, seine zwei Teile zeigend, einen armen an Plasma und einen reichen. Vergr. 500.

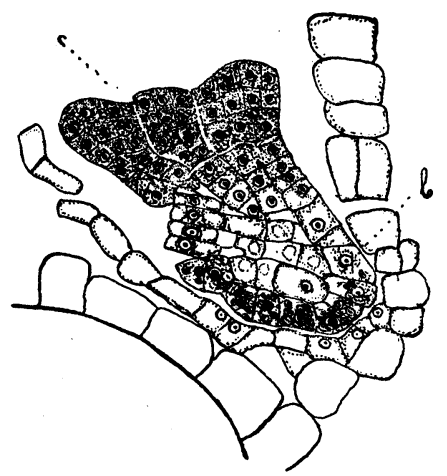

Fig. 43. Eins von den späteren Stadien des Embryos. $a$ Haustoriumzelle; $b$ Leitgewebe; $c$ Meristem. Vergr. 250.

Weitere Entwicklungsstadien sind in Fig. 39 u. 40 gegeben. Hier sieht man deutlich den Unterschied zwischen den zwei Hälften des Embryos, jener, welcher den Vegetationspunkt des Stämmchens und das primäre Blatt gibt, und der anderen, welche zum Haustoriumfuß wird. Die folgenden Entwicklungsschritte des Embryos sind in Fig. 34 u. 41 im Längsschnitt und in Fig. 42 von oben gesehen dargestellt. Endlich ist in Fig. 43 ein genügend formierter Embryo zu sehen, in dem man drei verschiedene Gewebekomplexe differenziert findet. Der obere Teil besteht aus embryonären Zellen (c), der untere hat den Charakter eines Haustoriums (a), seine Zellen sind mit großen Kernen versehen und mit Stärke gefüllt; der mittlere Teil (b) wird zu einer Art Leitgewebe. Wenn, wie gewöhnlich, sich ein Embryo bildet, so kommen auch Fälle vor, wo zwei bis drei und sogar noch mehr Embryos zu einer ziemlich weitgreifenden Entwicklung gelangen. Bauke sah nie zwei Salvinia-Embryos entstehen, dafür aber gelang dies Hofmeister. Fig. 31 zeigt zwei Archegonien, beide mit Embryos; in Fig. 32 sieht man drei Embryos entstehen. Trotz dieser Polyembryonie nimmt gewöhnlich ein Embryo überhand, den ganzen Nährwert der Makrospore an sich ziehend. 


\section{Einige Versuche über die Keimung der Makrosporen.}

Da ich genügend lebendes Material hatte, versuchte ich einige Experimente anzustellen, um über den Zusammenhang von Prothallium, Embryo und Makrospore bei Salvinia ins klare zu kommen.

I. Vor allen Dingen lag es mir daran, eine Embryoentwicklung ohne Befruchtung $\mathrm{zu}$ erhalten. $\mathrm{Zu}$ diesem $\mathrm{Zwecke}$ wurden sorgfältig von Mikrosporangien gesonderte Makrosporangien in einem Termostat unter verschiedenen Temperaturen von $22-30^{\circ} \mathrm{C}$ kultiviert. Wurden die Makrosporangien ätherisiert und mit Chloralhydrat bearbeitet, so fiel das Resultat natürlich negativ aus, die Embryos kamen ohne Befruchtung nicht zur Entwicklung. Das war auch zu erwarten, wenn man der interessanten Angaben Strasburger's über Apogamie bei Marsilia

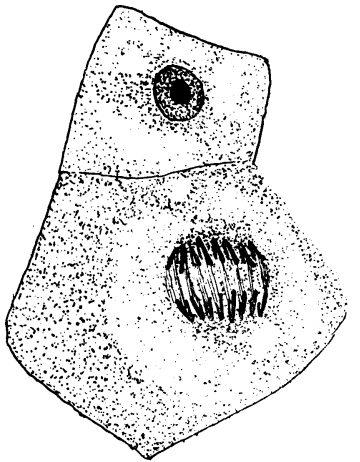

44
Fig. 44 u. 45 . Zwei Zellen mit Kernen auf gleichen Entwicklungsstadien.

Fig. 44 aus dem Embryo mit acht Chromosomen im Kern und aus dem Prothallium (Fig. 45) mit vier Chromosomen. Vergr. 1500.

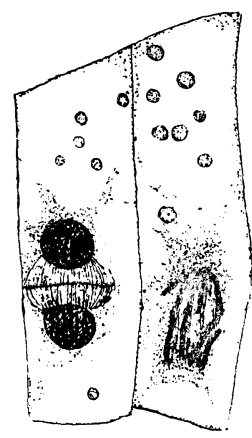

45

sich erinnert. Fig. 44 u. 45 zeigen die entsprechenden Teilungsstadien der Kerne im Prothallium (44) und im Embryo. Die Chromosomenzahl in den Kernen des Embryos beträgt 8, in den Kernen des Prothalliums nur 4 .

Bei den apogamen Arten von Marsilia ist die Zahl der Chromosomen sowohl im Protophyt als auch im Metaphyt dieselbe, wie die Beobachtungen Stras burger's beweisen. Die der Befruchtung entzogenen Makrosporen bildeten Prothallien mit einer großen Anzahl von Archegonien (viele Dutzende). Es ist interessant hier an die Zeichnungen Bauke's zu erinnern. Trotzdem aber, ungeachtet der langen Dauer der Kultur und den günstigen Bedingungen, erreichten die Prothallien nicht ihre normale Größe und entwickelten keine flügelartigen Fortsätze.

Diese Beobachtung brachte mich auf den Gedanken einer Abhängigkeit der Embryos von der Form des Prothalliums.

Dieselben Beobachtungen wiesen die merkwürdige Regelmäßigkeit nach, mit der sich die Archegonien nur auf der Seite ausbilden, wo 
das erste Archegonium angelegt ist, wo also noch längere Zeit das Meristem tätig ist. Wie schon aus den Versuchen Leitgeb's bekannt ist, werden die Archegonien in einer Abhängigkeit vom Lichte angelegt: sie entstehen auf der Schattenseite. Dies gilt aber nicht für Salvinia: ihre Archegonien werden gerade auf der vorderen oder oberen, also beleuchteten Seite angelegt. II. Um eine Archegonienentwicklung auch auf der anderen Seite hervorzurufen, stellte ich sie unter einen schwarzen Zylinder und ließ die Lichtstrahlen die Sporen von unten her treffen. Positive Resultate blieben aus. Eine große Anzahl Archegonien entwickelte sich, wie früher, auf der vorderen Seite; auf der hinteren Seite bildeten sich gleichfalls einzelne Archegonien, aber in sehr geringer Anzahl, wie aus den Schemen $46 A$ u. $B$ zu sehen ist.

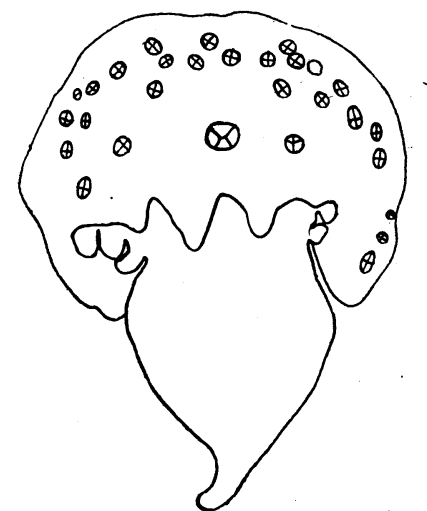

$46 \mathrm{~A}$

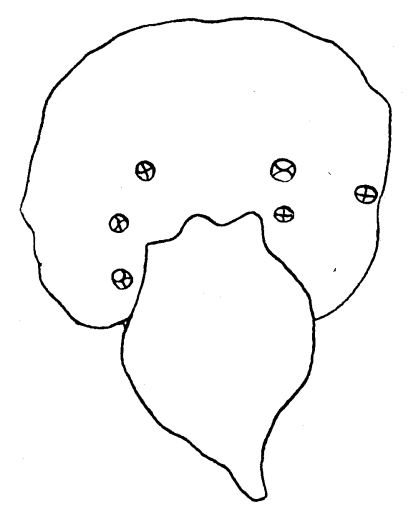

$46 B$

Fig. 46. $A$ Vorderseite des sterilen Prothalliums; $B$ Hinterseite desselben.

Größere Resultate bei Beleuchtung von unten erlangte ich nicht. Ich bin nicht geneigt anzunehmen, daß die Entstehung der Archegonien auf der hinteren Seite durch die Beleuchtung von unten bedingt war, umsomehr, als auch Bauke unter gewöhnlichen Umständen einzelne Archegonien auf der Hinterseite vorfand.

Aus allem Gesagten folgt, daß das Licht für die Verteilung der Archegonien bei Salvinia ohne Bedeutung ist. Man kann noch weiter gehen und erklären, daß die Entstehung der Archegonien vom Lichte vollkommen unabhängig ist; nämlich, auch die in einer photographischen Dunkelkammer gekeimten Sporen entwickelten normale Prothallien. (Vgl. Goebel's Einleitung in die experimentelle Morphologie, pag. 6.) Das Wachstum und die Entwicklung der Prothallien und der Embryos ließ eher an andere Faktoren als an das Licht denken. Es wurden folgende Experimente angestellt: 
III. Ein Teil der Makrosporangien wurde nach Isolierung ausgesät, der andere Teil mit Mikrosporangien gemischt. Der erste Teil entwickelte breite Prothallien mit einer Masse von Archegonien; die flügelartigen Fortsätze blieben in allen Fällen aus. Dagegen entwickelten die befruchteten, Embryos enthaltenden Prothallien, jene langen Flügel, welche jeder, der nur Salvinia $ᄋ$ Prothallien gesehen hat, kennt. Daraus folgt nun, daß die Form und das Wachstum des Prothalliums durch die Entwicklung des Embryos bedingt wird. Fehlt der Embryo, so wird das Meristem zur gesteigerten Produktion der Archegonien verbraucht. Wenn aber aus dem befruchteten Archegonium ein Embryo hervorgeht, so wird das Meristem zum Gewebe des Prothalliums.

IV. Um das zu beweisen wurde folgendes Experiment angestellt. Es wurden weibliche Prothallien genommen, an denen eine große Anzahl von Archegonien angelegt war, worunter die an den Seitenrändern befindlichen erst im Entstehen begriffen waren. $\mathrm{Zu}$ diesen $\bigcirc$ Prothallien wurde eine große Anzahl von Mikrosporangien, die es schon zur Antherozoidbildung brachten, zugesetzt. Als das Resultat dieser Vermischung war die Befruchtung einiger Randarchegonien. Danach fing das Meristem des Prothalliums an das Gewebe der Randseite auszuarbeiten, obgleich es zu einer vollkommenen Ausbildung derselben

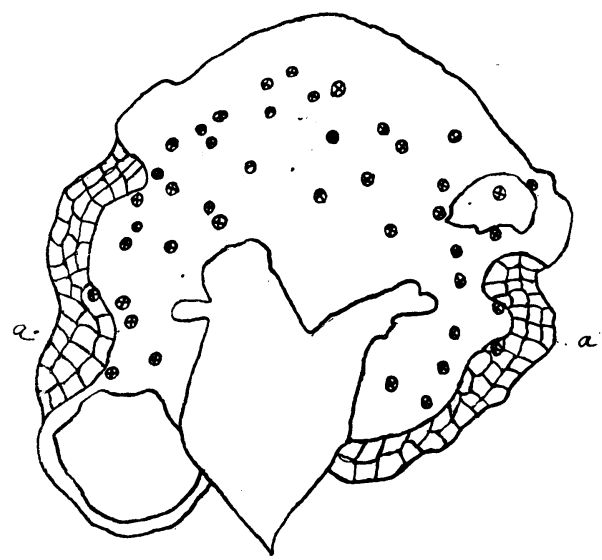

Fig. 47. Prothallium mit zwei Embryos, die aus den Rand-Archegonien stammen. Meristem sichtbar. $a$ u. $a$. nicht kam, da die Teilungsfähigkeit des Meristems so gut wie erschöpft war. Fig. 47 stellt solch ein Prothallium dar, dessen zwei Randarchegonien befruchtet waren und eben anfangen Embryos zu entwickeln. Der durch Zellen bezeichnete Teil des Prothalliums ist erst nach der Befruchtung zur Entfaltung gebracht. Diese Erscheinung wurde mehrere Male geprüft und jedesmal fiel das Resultat gleich aus. Also sind die Form des Prothalliums und die Erzeugung des Embryos - Korrelationserscheinungen.

V. Um in die Erscheinung besser einzudringen, war folgendes Experiment nötig: Es wurden von Mikrosporangien isolierte und eine 
Reihe von Archegonien bildende Prothallien von den sie tragenden Makrosporen abgetrennt und mit einer beträchtlichen Anzahl Antherozoiden erzeugender Mikrosporangien vermișcht. In dasselbe Gefäß wurden auch an den Makrosporen angeheftete Prothallien gesetzt. Nun geschah es, daß die Archegonien der normalen Prothallien auch normale Embryos gaben. Die abgeschnittenen Prothallien zeigten keinen einzigen Embryo. Die mehrfache Wiederholung des Experiments führte zu demselben Resultate. Trotz der großen Anzahl der schwärmenden Antherozoiden unterblieb die Befruchtung in den abgeschnittenen Prothallien und nach 3-4 Tagen sanken sie auf den Boden des Gefäßes, um bald abzusterben. Daraus sieht man, wie unentbehrlich für die Befruchtung die Anwesenheit der Makrosporen ist. Die Makrospore spielt dabei die Rolle eines hydrostatischen Apparates, der das Prothallium auf dem Niveau der obersten Wasserschicht emporhält. Man kann sogar bemerken, daß seine Oberfläche mit dem Wasserniveau in einer Linie liegt.

VI. Das grüne Prothallium von Salvinia führt in seinen Zellen Stärkekörner. Man könnte sich denken, daß auf ihm ein Embryo ohne die Teilnahme der Makrospore entstehen könne. $\mathrm{Zu}$ diesem Zwecke wurden Prothallien mit kaum entwickelten Embryos von den Makrosporen abgeschnitten und zusammen mit normalen Prothallien sich selbst überlassen. Kein einziger von den abgeschnittenen Prothallien hatte die Kraft, trotz dem Besitze von Chlorophyll, einen Embryo entstehen zu lassen. Die Anwesenheit der Makrospore und der in ihr aufgespeicherten Nährmaterialien ist unbedingt nötig für die Embryoentwicklung.

VII. Um dies zu beweisen wurde vom Prothallium, das bereits einen Embryo ausgebildet hatte, der größte Teil des Gewebes entfernt, außer den Zellen, die sich in der Nachbarschaft des entwickelten Embryos befanden; der Embryo gelangte zur normalen Ausbildung. Dieser Versuch zeigte gleichzeitig die Unfähigkeit des Prothalliums zu regenerieren. Außer den obengenannten wurden noch andere Experimente vorgenommen, z. B. mittels des Klinostaten usw., aber sie gaben keine zuverlässigen Resultate. Übrigens sind sie noch nicht beendigt. Einstweilen erwähne ich noch einen letzten Versuch, der mit auf Lehm gesäten Sporen ausgeführt war.

VIII. Auf dem Lehmboden unter Glasglocke ging die Prothalliumbildung ebenso erfolgreich vor sich, wie im Wasser. Es entstanden sehr hübsche Prothallien mit normalen Archegonien und Embryos. 
Aus allen diesen Versuchen ist folgender Schluß zu ziehen: Das Prothallium von Salvinia ist nichts weniger als eine unabhängige Bildung, trotz der täuschenden Anwesenheit von Chlorophyll in seinen Zellen. Es ist eben nur ein Organ der Makrospore, wie bei Marsilia und sogar Selaginella und Isoëtes, das nur dazu dient die Archegónien $\mathrm{zu}$ tragen, aber unfähig ist die wachsende sporentragende Generation zu ernähren.

November 1908, Botanisches Institut Charkow.

\section{Literatur.}

Arnoldi, Die Entwicklung des weiblichen Vorkeims bei den heterosporen Lycopodiaceen. Bot. Ztg. 1896.

Bauke, Einige Bemerkungen über das Prothallium von Salvinia natans. Flora 1879. Belajeff (I), Über die männlichen Prothallien der Hydropleriden. Bot. Ztg. 1898. Ders. (II), Über den Nebenkern in spermatogenen Zellen und die Spermatogenese bei den Farnkräutern. Ber. d. Deutsch. bot. Gesellsch. 1897.

Ders. (III), Über die Spermatogenese bei den Schachtelhalmen. Ibid., 1897.

Ders. (IV), Über die Cilienbildner in den spermatogenen Zellen. Ibid., 1898.

Ders. (V), Über die Centrosomen in den spermatogenen Zellen. Ibid., 1899.

Boleter (I), Fegatella conica Corda. Beihefte zum Bot. Zentralbl. 1901, XVIII.

Campbell (I), Mosses and Ferns. I. and II. Edition.

Ders. (II), Studies on the Ophioglossaceae. Annales du Jardin Buitenzorg 1907.

Davis (I), Sporeformation in Derbesia. Annals of Botany 1908.

Escoyez (I), Blépharoplaste et Centrosome dans le Marchantia polymorpha. La Cellule 1907, XXIV.

Goebel (I), Organographie, Pars II.

Ders., Einleitung in die experimentelle Morphologie, 1908.

Hofmeister (I), Vergleichende Untersuchungen 1851.

Ikeno (I), Die Spermatogenese von Marchantia polymorpha. Beiheft z. Bot. Zentralbl. 1903.

Ders. (II), Zur Frage nach der Homologie der Blepharoplasten. Flora 1906.

Yamanouchi (I), Spermatogenesis, Oogenesis and Fertilization in Nephrodium. Bot. Gazette 1908. (Hier umfangreiche Literaturangaben.)

Leitgeb (I), Über Bilateralität der Prothallien. Flora 1879.

Prantl (I), Zur Entwicklungsgeschichte des Prothalliums von Salvinia natans. Bot. Ztg. 1879.

Pringsheim (I), Zur Morphologie der Salvinia natans. Jahrb. f. wissensch. Bot., III, 1863. Gesammelte Abhandl., II.

Shaw (I), The fertilization of Onoclea. Annals of Botany 1898.

Ders. (II), Über die Blepharoplasten bei Onoclea und Marsilia. Ber. d. Deutschen bot. Gesellsch. 1898.

Treub (I), Études sur les Lycopodiacées. Annal. du Jardin Buitenzorg 1889-1890. Strasburger, Botanisches Praktikum und Lehrbücher. 